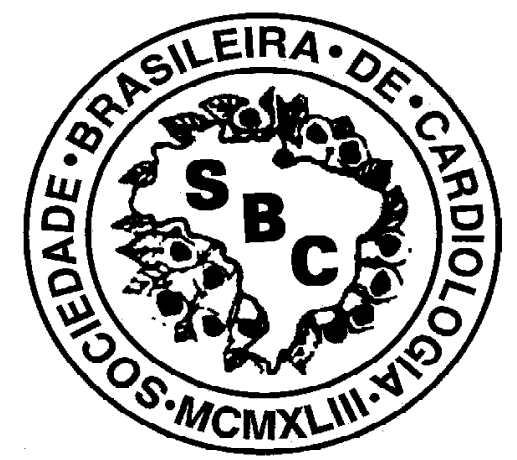

Diretriz de Indicações e Utilizações das Intervenções Percutâneas e Stent Intracoronariano na Prática Clínica 


\title{
Diretriz de Indicações e Utilizações das Intervenções Percutâneas e Stent Intracoronariano na Prática Clínica
}

\author{
Coordenador \\ Jorge Ilha Guimaraes \\ Editores \\ J. Eduardo Sousa (SP) \\ Expedito Ribeiro(SP) \\ Editores Associados \\ Luiz Alberto Mattos (SP) \\ Amanda GRM Sousa (SP) \\ Gilberto Lahorgue Nunes (RS) \\ Rogério Gomes Sarmento Leite (RS) \\ Participantes \\ Álvaro Vieira Moura(PR) \\ Carlos A. Mascia Gottschall (RS) \\ Eduardo A. da Silva Costa (PA) \\ Francisco C. Couto Falcão (CE) \\ Mauricio Lopes Prudente (GO) \\ Raimundo João Costa Furtado (MA)
}


Arq Bras Cardiol

volume 80, (suplemento I), 2003

\section{I- Introdução}

A intervenção coronariana percutânea é um método estabelecido e utilizado, com freqüência, na revascularização do miocárdio em portadores da doença arterial coronariana. Desde a sua introdução, por Gruentzig e cols, em $1977^{1}$, inúmeros dispositivos foram sendo testados na prática clínica, visando aprimorar os resultados inicialmente obtidos com o balão, ou seja, reduzir as complicações imediatas (oclusão do vaso, infarto do miocárdio e realização de cirurgia) e tardias (reestenose e nova revascularização) ${ }^{2,3}$.

Os stents coronarianos tornaram-se o método percutâneo de escolha preferencial, tanto pela maior segurança do procedimento, quanto pela diminuição das taxas de reestenose em relação aos demais dispositivos anteriormente testados. Inúmeros estudos controlados comprovaram tal superioridade, nas mais diversas apresentações clínicas e angiográficas da doença arterial coronariana $a^{4-7}$.

Estas diretrizes objetivam revisar, de maneira crítica, o papel e as indicações das intervenções percutâneas e dos stents coronarianos no tratamento de pacientes com doença coronariana. Para tanto, foi empregada a classificação das evidências de acordo com o formato proposto pelo American College of Cardiology/American Heart Association, na forma que segue:

Classe I: Condições em que existem evidências e/ou concordância geral de que o procedimento ou tratamento é benéfico, útil e efetivo.

Classe II: Condições em que existem evidências conflitantes e/ou divergências nas opiniões sobre a utilidade e eficácia do procedimento ou tratamento.

Classe IIa: Condições em que o peso das evidências e opiniões favorece a utilidade e eficácia do procedimento ou tratamento.

Classe IIb: Condições em que a utilidade e eficácia do procedimento ou tratamento não estão muito bem estabelecidas por evidências e opiniões.

Classe III: Condições em que existem evidências e/ou concordância geral de que o procedimento ou tratamento não é benéfico ou útil, podendo até ser deletério.

Nível de evidência A: dados oriundos de múltiplos ensaios clínicos randomizados

Nível de evidência $\boldsymbol{B}$ : dados oriundos de um único ensaio clínico randomizado ou estudos não randomizados

Nível de evidência $C$ : opiniões consensuais de especialistas no assunto.

\section{II- Considerações gerais}

A) Definição de sucesso - O sucesso do procedimento pode ser avaliado de modo angiográfico e clínico.
Diretriz de Indicações e Utilizações das Intervenções Percutâneas e Stent Intracoronariano na Prática Clínica

1. Angiográfico: após a realização da angioplastia com o balão, redução da estenose coronariana para $<50 \%$, com fluxo TIMI-3. Com o implante destents, recomenda-se a redução da estenose para $<20 \%$, também com fluxo epicárdico coronariano normal (TIMI-3) ${ }^{8}$.

2. Clínico: sobrevida com boa evolução clínica imediata, livre da ocorrência de infarto do miocárdio (novas ondas Q), de nova revascularização para o vaso-alvo (percutânea e cirúrgica). Com a progressiva utilização de novos dispositivos de revascularização percutâneos, é possível detectar elevação enzimática $(>3 x$ até $<5 x)$, sem alteração eletrocardiográfica ou surgimento de sintomas até $20 \%$ dos casos. Seu significado e sua incidência são motivos de discussão. É um consenso que elevações até 3 vezes o valor normal não promovem modificação prognóstica tardia; contudo, acima desse valor e, certamente, maior que 5 vezes, existem implicações prognósticas negativas tardias. Sua elevação é mais freqüente em procedimentos que associam o implante de stents com técnicas ateroablativas, manejo de enxertos de veia safena, pacientes com angina instável Classe IIIB (Braunwald). Sua verificação isolada não é considerada insucesso do procedimento ${ }^{8-11}$, porém elevações $>3$ vezes em pacientes com sintomas de infarto ou que tenham apresentado complicações angiográficas durante o procedimento (oclusão aguda, perda de ramo lateral importante ou slow-flow) devem ser consideradas como infarto significativo e, conseqüentemente, insucesso clínico ${ }^{8}$.

B) Graduação da gravidade das lesões - Uma nova classificação foi proposta em 2001, pela força-tarefa norteamericana, adaptando-se à nova era de dispositivos percutâneos ${ }^{2,8}$. (quadro I).

C) Fatores prognósticos de maior adversidade-Diversas variáveis clínicas foram relacionadas a um maior potencial de insucesso e reconhecidas para a ocorrência de desfechos adversos, os quais foram coletados de diversos estudos consecutivos e ensaios randomizados. As variáveis

Quadro I - Classificação das lesões coronarianas de acordo com o National Cardiovascular Data Registry.

Lesão de baixo risco

Focal $(<10 \mathrm{~mm})$ : concêntrica, acesso fácil, ângulo $<45^{\circ}$, contornos lisos, calcificação discreta ou ausente, lesão não-oclusiva, não-ostial, sem ramo adjacente envolvido e sem trombo.

Lesão de moderado risco

Tubular (10 até $20 \mathrm{~mm}$ de extensão): excêntrica, tortuosidade moderada, ângulo $>45^{\circ}$ até $<90^{\circ}$, contorno irregular, calcificação moderada, oclusão crônica ( $<3$ meses), lesão ostial, estenose em ramo que necessite duplo-guia e com algum grau de trombo presente.

Lesão de alto risco

Difusa $(>20 \mathrm{~mm})$ : grande tortuosidade, ângulo $>90^{\circ}$, oclusão crônica (>3 meses) ou presença de colaterais em "medusa", incapacidade de proteger ramo adjacente com lesão presente e enxerto de veia safena degenerado. 
que exibiram, em mais de $50 \%$ dos estudos analisados, risco $\geq 2$ vezes estão listadas no quadro II $^{8}$.

D) Experiência da instituição e do operador - A constante atualização do binômio hospital-operador é necessária, tanto com referência à modernização dos equipamentos, treinamento e atualização dos técnicos e enfermeiras, como do médico-operador. Instituições com maior volume exibem resultados melhores, mesmo em se tratando de pacientes de mais alto risco. As recomendações devem seguir os padrões normativos internacionais, baseadas em análise de estudos relacionados à aferição dos resultados, de acordo com o número de procedimentos hospital-operador ${ }^{8}$.

Classe I - Procedimentos realizados em centros com número anual $>400$ e operador que faça $\geq 75$ casos ao ano (nível de evidência $B$ ).

Classe II - Procedimentos realizados em centros com volume $>200$, porém $<400$ casos ao ano, com operador que realize $\geq 75$ casos anuais; ou hospitais com mais de 400 casos por ano, mas operador com $<75$ procedimentos anuais. Recomenda-se, de forma ideal, que esses operadores trabalhem em hospitais com mais de 600 casos/ano (nível de evidência C).

Classe III - Procedimentos em hospitais com volume menor ( $>200$ até $<400$ casos/ano) e operador com baixo volume anual ( $<75$ casos). Recomenda-se que os hospitais que realizem menos de 200 procedimentos anuais reavaliem cuidadosamente o seu programa de intervenção coronariana percutânea, determinando, de maneira criteriosa e independente, os níveis de sucesso e as taxas de complicações, e a conveniência ou não de manter o serviço operando, especialmente no caso de este hospital não ser centro de referência regional (nível de evidência C).

E) Intervenção coronariana percutânea e cobertura cirúrgica - Apesar dos avanços tecnológicos observados com o uso de stents, outros dispositivos e a nova farmacoterapia antiplaquetária, a realização de casos eletivos em centros hospitalares sem um serviço de cirurgia cardíaca atuante não é recomendada ${ }^{8}$.

Quadro II - Variáveis clínicas relacionadas ao pior prognóstico em pacientes submetidos à intervenção coronariana percutânea.

Idade $\geq 75$ anos; sexo feminino; fração de ejeção <40\%; angina estável (Classe IV); angina instável; infarto do miocárdio $(<24 \mathrm{~h})$; infarto do miocárdio prévio recente ( $>1$ até $<7$ dias); insuficiência cardíaca congestiva; choque cardiogênico; procedimento de emergência (pós-ressuscitação cardíaca); doença arterial periférica importante; acidente vascular cerebral prévio; uso de balão intra-aórtico prévio; valvopatia grave (área aórtica $\leq 1,0 \mathrm{~cm}^{2}$ e ou regurgitação mitral $>2 \mathrm{x}$ ); creatinina $>2,0 \mathrm{mg} / \mathrm{dl}$; diálise; lesão de alto risco; lesão no tronco da coronária esquerda (protegido ou não); procedimento para enxerto de veia de safena e lesões com trombo
A exceção está relacionada ao procedimento percutâneo primário, isto é, àquele utilizado como método de reperfusão coronariana mecânica nas primeiras horas do início do infarto do miocárdio. Esse é um posicionamento mais recente, em vista da possibilidade de ampliar o número de pacientes tratados. Essa hipótese foi verificada por alguns estudos (Air-PAMI e C-Port), com bons e seguros resultados iniciais ${ }^{12-16}$.

Classe I-Procedimentos eletivos e primários no infarto do miocárdio realizados em centros com serviço de cirurgia cardíaca (nível de evidência B).

Classe IIa -Procedimentos primários (infarto do miocárdio com supradesnível do segmento ST) realizados em centros sem serviço de cirurgia cardíaca; efetuados, contudo, por operador experiente ( $>35$ intervenções primárias no IAM/ano) e com possibilidade de completo suporte hemodinâmico durante a transferência emergencial para um serviço de cirurgia cardíaca próximo ( $<1$ hora) (nível de evidência A).

Classe III - Procedimentos eletivos em hospitais sem serviço de cirurgia cardíaca ou realizados no IAM sem um plano de transferência para um serviço próximo com cirurgia cardíaca disponível (nível de evidência C).

\section{III- Intervenção coronariana percutânea no Brasil}

O exponencial crescimento da prática da intervenção coronariana percutânea no Brasil está documentado por meio do registro CENIC (Central Nacional de Intervenções Cardiovasculares), órgão oficial da SBHCI (Sociedade Brasileira de Hemodinâmica e Cardiologia Intervencionista) que, desde 1991, contabiliza os procedimentos percutâneos cardiovasculares realizados em nosso país. No biênio 1992/93, 16.429 pacientes foram submetidos ao procedimento, todos por meio do balão. A análise do biênio 1996/ 97 demonstra um crescimento de $33 \%$ na realização dos procedimentos percutâneos. Foram, então, tratados 24.667 pacientes, sendo $44 \%$ dos procedimentos já com o implante dos stents coronarianos. No biênio seguinte, 1999/2000, o crescimento foi ainda maior, de 37\%, com 39.469 enfermos submetidos à revascularização miocárdica percutânea, tendo a maioria dos procedimentos empregado os stents $[\mathrm{n}=27.502(69,7 \%)]$. Neste último biênio, 11.744 pacientes ainda utilizaram apenas o balão e, em apenas $223(0,6 \%)$ dos casos restantes, foi necessário outro dispositivo percutâneo (aterótomos ou laser). A supremacia dos stents coronarianos fica assim bem caracterizada. A melhoria observada nos resultados imediatos, com maior sucesso e redução nas complicações, está evidenciada na tabela $\mathrm{I}^{3,17,18}$. 
Tabela I - Evolução temporal da prática clínica da intervenção coronariana percutânea no Brasil, considerando a avaliação do uso de stents coronarianos e os resultados imediatos, de acordo com o registro CENIC/SBHCI.

\begin{tabular}{|c|c|c|c|c|c|}
\hline Ano & 1997 & 1998 & 1999 & 2000 & $\mathrm{p}$ \\
\hline Vasos tratados & 16.000 & 18.070 & 20.677 & 27.665 & $<0,001$ \\
\hline Uso de stents & $44,6 \%$ & $56,2 \%$ & $63,6 \%$ & $76,7 \%$ & $<0,001$ \\
\hline Sucesso & $92,6 \%$ & $94,3 \%$ & $95,1 \%$ & $96,1 \%$ & $<0,001$ \\
\hline Estenose final & $19,1 \pm 22,2 \%$ & $16,8 \pm 19,9 \%$ & $16,0 \pm 16,2 \%$ & $11,7 \pm 17,6 \%$ & $<0,001$ \\
\hline Oclusão aguda & $2,0 \%$ & $1,4 \%$ & $1,0 \%$ & $0,8 \%$ & $<0,001$ \\
\hline IAM (onda Q) & $1,6 \%$ & $1,6 \%$ & $1,1 \%$ & $0,6 \%$ & $<0,001$ \\
\hline Cirurgia de urgência & $0,5 \%$ & $0,5 \%$ & $0,15 \%$ & $0,15 \%$ & $<0,001$ \\
\hline Óbito hospitalar & $1,6 \%$ & $1,6 \%$ & $1,3 \%$ & $1,2 \%$ & 0,002 \\
\hline
\end{tabular}

\section{IV - Recomendações para a técnica de implante do stent coronariano}

Como demonstrado no registro CENIC/SBHCI, o implante dos stents tornou-se o padrão de dispositivo utilizado para a realização da intervenção coronariana percutânea. Algumas recomendações devem ser observadas, para a obtenção de um resultado imediato e tardio considerado ótimo.

Em 1995, Colomboe cols modificaram a técnica inicialmente utilizada para o implante de stents coronarianos, baseado nos achados do ultra-som intracoronariano ${ }^{19}$. Cateteres-balão de alta pressão (>12 atm) foram utilizados para melhorar os resultados iniciais, após a identificação, por meio do ultra-som, dos resultados subótimos; tendo sido também promovida a modificação do protocolo farmacológico ${ }^{20,21}$. Inúmeras experiências foram apresentadas mundialmente após esses estudos iniciais, confirmando e reproduzindo esses resultados. O conceito do implante ótimo foi incorporado à prática rotineira.

As recentes melhorias no material empregado, assim como na terapeutica adjunta antiplaquetária propiciaram a obtenção de um implante ótimo de stents coronarianos, na imensa maioria dos pacientes tratados (>95\%), mesmo frente a quadros clínico e angiográfico julgados como adversos no passado, seja a isquemia miocárdica silenciosa ou o tratamento do infarto agudo do miocárdio comstents; sejam os vasos mais finos ou as lesões mais longas.

A grande maioria dos stents coronarianos utilizados no Brasil é pré-montada. Com os sistemas modernos de fixação do stent, a plataforma de expansão e o cateterbalão, pode-se impedir, com segurança, a sua liberação em local inadequado, ou a liberação inadvertida na corrente sangüínea. Os sistemas de baixo perfil predominam, o que permite o seu implante sem necessidade de pré-dilatação $0^{22,23}$. A redução do calibre dessas modernas plataformas, associada à ampliação da luz dos cateteres-guia, permite o emprego de introdutores de calibre reduzido, a partir de 5 French.

Recomenda-se a angioplastia com o cateter-balão adicional, seja com a própria plataforma do stent utilizada, seja com o uso de balões adicionais, de diâmetro crescente (relação balão/artéria $\geq 1: 0-1: 1)$, atingindo-se pressões $\geq 12$ atm. As altas pressões, com relação balão-artéria $>1: 1$, implicam maior risco de perfuração, principalmente em vasos de diâmetro $\leq 3,0 \mathrm{~mm}$, estenoses de coronária direita e pacientes do sexo feminino ${ }^{24}$.

Um obstáculo para a obtenção do implante ótimo está relacionado à presença de calcificação coronariana, impedindo o acesso dos stents ao local desejado. Se, mesmo após o emprego de cateteres-guia e fios-guia de suporte máximo, fracionamento da extensão dos stents, maior número de stents implantados com extensão mais curta, não for obtida a solução do problema, deve-se discutir o envio do paciente para cirurgia coronariana em caráter de emergência. Ojulgamentoé individual, a partir de análises da quantidade e viabilidade do miocárdio em risco, do grau de estenose residual $(>50 \%)$, da presença de dissecções ou estenoses graves, não completamente cobertas comstents, e até de situações mais adversas, como distúrbio de fluxo coronariano e trombos associados. Pacientes com grandes áreas de miocárdio viável, com estenose residual $>50 \%$ e estenose e/ou dissecções não corretamente solucionadas percutaneamente devem ser encaminhados para a cirurgia. O risco de oclusão aguda ou subaguda é elevado. Essa sinergia de adversidades, com um desfecho para um evento maior (cirurgia de emergência), é muito pouco freqüente, incidindo em menos de $0,5 \%$ dos pacientes tratados.

Classe I - Implante de stent coronariano com alta pressão de liberação ( $\geq 12 \mathrm{~atm})$, associado à prescrição prévia de aspirina ( $200 \mathrm{mg} / \mathrm{VO}, 24 \mathrm{~h}$ antes $)$ e ticlopidina ( $500 \mathrm{mg} / \mathrm{VO}$ (ataque) e $250 \mathrm{mg} / \mathrm{VO} 2 \mathrm{x} / \mathrm{dia}, 48 \mathrm{~h}$ antes) ou clopidogrel ( $300 \mathrm{mg} / \mathrm{VO}$ (ataque) e $75 \mathrm{mg} / \mathrm{VO}, 48 \mathrm{~h}$ antes) (nível de evidência A).

A) Monitoração doprocedimento-No momento atual, não existe uma recomendação formal para utilização, de forma sistemática, como método auxiliar no implante dos stents, do ultra-som intracoronariano, de sistemas de medida de gradiente translesional (pressure-wire) ou de medida 
da reserva de fluxo coronariano (Dopplerflow-wire). Diversos estudos randomizados e séries demonstram que os resultados imediatos (sucesso angiográfico ou ocorrência de eventos adversos maiores) foram similares, quer tenham sido empregadas essas técnicas adjuntas ou a angiografia coronariana quantitativa ${ }^{25-28}$. Existe controvérsia em relação à possível redução das taxas de reestenose intra-stent, com a monitoração do implante por meio do ultra-som intracoronariano, evidências que estão em discussão nas diretrizes específicas $^{29-34}$ (Classe IIb, nível de evidência A).

B) Farmacoterapia adjunta - A realização de uma angioplastia com o balão, assim como a utilização de dispositivos atero-ablativos necessitam apenas da administração de aspirina (200 mg/VO/dia) prévia, sendo que, em casos de procedimentos não planejados, sugere-se a mastigação do (s) comprimido (s) o mais breve possível.

O implante dos stents coronarianos requer a associação da aspirina com um tienopiridínico, seja a ticlopidina (250 mg/VO/2Xdia/30dias) ou o clopidogrel (300 mg/VOe 75 $\mathrm{mg} / \mathrm{VO} / \mathrm{dia} / 30$ dias). Essa associação forneceu as menores taxas de reoclusão imediatas dos stents ${ }^{35}$. A ticlopidina exige um pré-tratamento de, pelo menos, $48 \mathrm{~h}$ antes da realização do procedimento. O clopidogrel, de uso mais recente, exibe menor incidência de para-efeitos, como alergia, rash cutâneo e ocorrência de leucopenia ${ }^{35}$. Como vantagem adicional, sua dose de ataque $(300 \mathrm{mg} / \mathrm{VO})$ pode ser administrada minutos antes do procedimento, já fornecendo níveis sistêmicos razoavelmente adequados. Quanto à duração do tratamento, pode-se afirmar que, até o momento, os benefícios observados perduraram até o final do primeiro mês, não sendo formalmente recomendada a manutenção prolongada da sua administração ${ }^{20,21,35,36}$ (Classe I, nível de evidência A), a não ser em casos de braquiterapia e de emprego de stents farmacológicos.

A utilização sistemática de inibidores da glicoproteína IIb/IIIa é controversa. Diversos estudos demonstram a redução dos desfechos adversos combinados, sejam imediatos ou tardios, em pacientes submetidos à intervenção coronariana percutânea, com ou sem a administração desses fármacos (EPIC, EPILOG, EPISTENT e
ESPRIT). Contudo, a redução significativa mais acentuada ocorreu nos chamados "infartos enzimáticos" (diagnosticados apenas pela elevação dos marcadores séricos cardíacos). Na prática, recomenda-se sua utilização em pacientes submetidos ao procedimento na vigência de síndrome coronariana aguda (ver indicação clínica), principalmente na ausência de pré-tratamento com um tienopiridínico. Em procedimentos eletivos, independentemente da complexidade, sua indicação é ainda questionada, com a possível exceção dos pacientes diabéticos ${ }^{37-40}$ (Classe IIa, nível de evidência $A$ ).

Recentemente, um estudo randomizado e placebo controlado em pacientes com níveis médios de colesterol (entre 135 e $270 \mathrm{mg} / \mathrm{dl}$ ), submetidos a implante destent coronariano, mostrou que o tratamento com fluvastatina $80 \mathrm{mg} /$ dia (iniciado por ocasião da alta hospitalar e mantido por, no mínimo, 3 anos) reduziu em $22 \%$ a ocorrência de eventos cardíacos maiores (morte e infarto) na evolução tardia ${ }^{41}$.

\section{V-Indicações clínicas}

Pacientes com anatomia favorável podem ser tratados percutaneamente, independentemente do grau e da extensão da doença arterial coronariana, com baixo risco de complicações $(<2 \%)$ e altas taxas de sucesso ( $>95 \%)$. Dessa forma, as indicações atuais para o implante dos stents devem basear-se mais nos dados anatômicos (factibilidade) das lesões a serem tratadas, englobando todo o espectro de apresentações clínicas da doença arterial coronariana, desde a isquemia silenciosa até o infarto do miocárdio ${ }^{8,42}$.

Classe I-Pacientes com isquemia assintomática, angina classe I a IV (Canadian Cardiovascular Society) ou instável, com expressiva área de miocárdio viável, com uma ou mais lesões significativas, em um ou mais vasos coronarianos, com anatomia favorável, alta possibilidade de sucesso e baixo risco de morbidade e mortalidade (nível de evidência A).

Classe II a -O mesmo espectro clínico e anatômico da classe I, exceto se o paciente for diabético ou apresentar área de miocárdio em risco viável de tamanho moderado (nível de evidência $B$ ).

\begin{tabular}{|c|c|c|c|c|}
\hline & \multicolumn{2}{|c|}{$\begin{array}{l}\text { Nova revascularização vaso-alvo } \\
\text { Diabético / Não-diabético }\end{array}$} & \multicolumn{2}{|c|}{$\begin{array}{c}\text { Reestenose } \\
\text { Diabético / Não-diabético }\end{array}$} \\
\hline & Balão & Stent & Balão & Stent \\
\hline Van Belle ( $\mathrm{n}=113 / 487)$ & & & $63 \% / 36 \% *$ & $25 \% / 27 \%$ \\
\hline Abizaid (n=195/560) & - & $17,7 \% / 9,1 \% *$ & - & \\
\hline Elezi $(n=715 / 2,839)$ & - & $21,1 \% / 15,6 \% *$ & - & $37,5 \% / 28,3 \% *$ \\
\hline Chaves $(n=321 / 1,443)$ & - & $16,9 \% / 11,7 \% *$ & & \\
\hline Mattos $(n=135 / 758)^{*}$ & $13 \% / 14 \%$ & $14 \% / 7 \% *$ & $32 \% / 33 \%$ & $36 \% / 19 \% *$ \\
\hline
\end{tabular}


Arq Bras Cardiol

volume 80, (suplemento I), 2003

Classe II b - Pacientes com isquemia assintomática ou angina discreta e área pequena de miocárdio viável em risco (nível de evidência B).

Classe III - Pacientes com as mesmas características clínicas anteriormente citadas, porém sem evidência de isquemia miocárdica, com pequena área de miocárdio viável, sintomas atípicos que não sugiram isquemia miocárdica, fatores associados com risco aumentado de morbi-mortalidade, doença de tronco da coronária esquerda (não protegido) ou estenose não-significativa $(\leq 50 \%)$ (nível de evidência A).

A) Diabético - Os pacientes diabéticos permanecem como um dos subgrupos de maior dificuldade para o tratamento percutâneo ${ }^{43-45}$. Os stents coronarianos exibem indicação preferencial em relação ao balão, como evidenciado na tabela II, apesar de alguma controvérsia observada nas séries já apresentadas ${ }^{46-50}$. Existem variáveis clínico-angiográficas que facilitam a indicação do melhor procedimento para a revascularização do miocárdio. Entre os diabéticos, são considerados melhores candidatos para a intervenção percutânea (Classe II a, nível de evidência A) os que apresentam vaso-alvo com diâmetro de referência $\geq 3,0 \mathrm{~mm}$, extensão da lesão-alvo $\leq 25 \mathrm{~mm}$, ausência de microalbuminúria e lesão uniarterial.

De forma oposta, a revascularização cirúrgica tem preferência no caso de diabéticos, frente às seguintes variáveis: múltiplos vasos $(\geq 2)$, associados ou não à estenose no tronco da coronária esquerda; estenoses longas (>25 $\mathrm{mm})$ com vasos de diâmetro reduzido $(<3,0 \mathrm{~mm})$; vaso derradeiro com grande área de miocárdio em risco; disfunção ventricular grave; oclusão crônica, suprindo grande área de miocárdio viável; proteinúria associada no paciente diabético dependente de insulina.

A.1) Uso de abciximab: O uso sistemático de um inibidor da glicoproteína IIb/IIIa (abciximab) associado ao procedimento percutâneo é, ainda, controverso. Apenas uma subanálise de um estudo randomizado apresentou evidências favoráveis tardias, clínicas e angiográficas (redução de
Diretriz de Indicações e Utilizações das Intervenções Percutâneas e Stent Intracoronariano na Prática Clínica

mortalidade, perda luminal e nova revascularização do vaso-alvo após o implante do stent). Novos estudos são necessários para ratificar ou não sua indicação ${ }^{51}$ (Classe IIb, nível de evidência B).

A.2) Doença multiarterial: Em relação aos portadores de múltiplas lesões submetidos ao implante do stent coronariano, a subanálise do estudo randomizado ARTS (stents múltiplos vs. cirurgia de revascularização) avaliou os resultados, ao final de um ano, em 208 diabéticos. A mortalidade foi maior nos pacientes tratados com stents $(6,3 \%$ vs. $3,1 \% ; \mathrm{p}=0,29)$, e a ocorrência de acidente vascular cerebral, maior naqueles submetidos à cirurgia $(1,8 \%$ vs. $6,3 \% ; \mathrm{p}=0,09$ ), ainda que não tenha havido diferença estatisticamente significante. Por outro lado, uma nova revascularização foi bem mais comum nos enfermos tratados com implante do stent $(22,3 \%$ vs. $3,1 \%$; $\mathrm{p}<0,05)$. A indicação de um procedimento multiarterial em diabéticos deve ser personalizada, sendo preferencial no tratamento de enfermos portadores de lesões biarteriais ${ }^{52}$ (Classe IIb, nível de evidência B).

A nova tecnologia dosstents revestidos é muito promissora $^{53,54}$. O resultado da subanálise de diabéticos, incluídos no estudo randomizado RAVEL ( $\mathrm{n}=44 ; 0 \% \mathrm{vs}$. $42 \%$; $<<0,001$ ), que comparou o implante de um stent convencional ao stent revestido com sirolimus $\left(\mathrm{Cypher}^{\circledR}\right)$, evidenciou reduzida taxa de reestenose intra-stent, na cinecoronariografia de seis meses. Entretanto, como tais resultados foram observados em uma análise de subgrupo, envolvendo um pequeno número de pacientes, é necessária a sua confirmação em novos estudos clínicos, que avaliem, especificamente, o emprego do stent revestido em grupos de diabéticos, antes de serem feitas recomendações definitivas.

B) Síndrome coronariana aguda -B.1) Angina instável: Nos pacientes com angina instável, o tratamento clínico de "passivação" deve ser realizado previamente à intervenção; de forma precoce (18-48h), principalmente, nos pa-

\begin{tabular}{|c|c|c|c|c|}
\hline \multicolumn{5}{|c|}{ Tabela III - Escore de risco do TIMI } \\
\hline \multirow[t]{2}{*}{ História } & \multirow[t]{2}{*}{ Pontos } & \multicolumn{3}{|c|}{ Risco de Eventos Cardíacos em 14 dias (\%) } \\
\hline & & Escore de risco & $\begin{array}{l}\text { Morte ou } \\
\text { infarto }\end{array}$ & $\begin{array}{c}\text { Morte, infarto ou } \\
\text { revascularização urgente }\end{array}$ \\
\hline Idade $>65$ anos & 1 & & & \\
\hline$\geq 3$ fatores de risco para doença coronariana & 1 & $0 / 1$ & 3 & 5 \\
\hline Doença coronariana conhecida (estenose $\geq 50 \%$ ) & 1 & 2 & 3 & 8 \\
\hline Uso de AAS nos últimos 7 dias & 1 & 3 & 5 & 13 \\
\hline Apresentação & & 4 & 7 & 20 \\
\hline Angina recente $(\leq 24 \mathrm{~h})$ e severa & 1 & 5 & 12 & 26 \\
\hline Elevação de marcadores cardíacos & 1 & $6 / 7$ & 19 & 41 \\
\hline Desvio do segmento $\mathrm{ST} \geq 0,5 \mathrm{~mm}$ & 1 & & & \\
\hline Escore de risco $=$ total de pontos & (0 a 7$)$ & & & \\
\hline
\end{tabular}


Diretriz de Indicações e Utilizações das Intervenções Percutâneas e Stent Intracoronariano na Prática Clínica
Arq Bras Cardiol

volume 80, (suplemento I), 2003

\begin{tabular}{|c|c|c|c|c|}
\hline \multicolumn{5}{|c|}{$\begin{array}{c}\text { Tabela IV - Resultados ao final de um ano dos estudos STENT-PAMI } \\
\text { e CADILLAC, comparando stent e balão utilizados de forma } \\
\text { primária no infarto do miocárdio. }\end{array}$} \\
\hline & \multicolumn{2}{|c|}{ STENT-PAMI $(n=900)$} & \multicolumn{2}{|c|}{ CADILLAC $(\mathrm{n}=2,082)$} \\
\hline & Balão & Stent & Balão & Stent \\
\hline Reinfarto & $2,7 \%$ & $2,9 \%$ & $2,9 \%$ & $2,2 \%$ \\
\hline Mortalidade & $3,0 \%$ & $5,4 \%$ & $4,3 \%$ & $4,4 \%$ \\
\hline Nova revascularização* & $21,0 \%$ & $10,6 \%$ & $17,6 \%$ & $9, \%$ \\
\hline Reoclusão* & $9,3 \%$ & $5,1 \%$ & $11,3 \%$ & $5,7 \%$ \\
\hline Reestenose* & $33,5 \%$ & $20,6 \%$ & $40,8 \%$ & $22,2 \%$ \\
\hline
\end{tabular}

cientes com moderado e alto risco (troponina positiva e escore TIMI >3, conforme tabela III, de acordo com ensaios recentes (FRISC-II e TACTICS-TIMI 18) ${ }^{55,56}$. Esses enfermos beneficiam-se da farmacoterapia adjunta, como uso de inibidor da glicoproteína IIb/IIIa (TACTIS-TIMI 18) e dos tienopiridínicos, como o clopidogrel (PCI-CURE), em emprego prévio à realização do implante do stent. A indicação do procedimento segue, então, os preceitos anteriores ${ }^{36,55,56}$ (Classe I, nível de evidência A).

B.2. Infarto do miocárdio: A intervenção coronariana percutânea primária, isto é, aquela praticada nas primeiras horas do início do infarto do miocárdio, sem o uso prévio de fibrinolíticos, já tem sua normatização clínica definida nas diretrizes clínicas desta síndrome ${ }^{12}$. O implante dos stents coronarianos de forma primária demonstrou resultados superiores ao do balão, principalmente em relação à redução da reestenose e da necessidade de realizar uma nova revascularização tardia, de maneira muito consistente, em dois estudos: STENT-PAMI e CADILLAC. Sua utilização, contudo, não modificou a ocorrência dos desfechos adversos principais relacionados ao infarto do miocárdio (tab IV). Realizar uma angioplastia primária, apenas com o balão, é admissível, porém é necessário obter um resultado angiográfico ótimo, isto é, fluxo coronariano normal (TIMI-3), estenose residual inferior a $30 \%$ e ausência de dissecção coronariana ou de trombos residuais. Caso contrário, o implante dos stents é obrigatório, visando à profilaxia da isquemia recorrente e consequiente reoclusão do vaso tratado (57-60) (Classe I, nível de evidência A).

\begin{tabular}{|c|c|c|}
\hline \multicolumn{3}{|c|}{$\begin{array}{l}\text { Tabela V - Efeito do implante do stent comparado à angioplastia com } \\
\text { o balão, de acordo com o diâmetro do vaso, nos estudos randomizados } \\
\text { STRESS e BENESTENT-I. }\end{array}$} \\
\hline \multirow[b]{2}{*}{ Diâmetro do vaso-alvo } & \multicolumn{2}{|c|}{ Reestenose coronariana } \\
\hline & Balão & $\overline{\text { Stent }}$ \\
\hline$<2,6 \mathrm{~mm}$ & $42 \%$ & $38 \%$ \\
\hline $2,6-2,8 \mathrm{~mm}$ & $49 \%$ & $27 \%$ \\
\hline $2,8-3,0 \mathrm{~mm}$ & $40 \%$ & $27 \%$ \\
\hline $3,0-3,4 \mathrm{~mm}$ & $34 \%$ & $18 \%$ \\
\hline$>3,4 \mathrm{~mm}$ & $30 \%$ & $22 \%$ \\
\hline
\end{tabular}

\begin{tabular}{|c|c|c|c|c|}
\hline \multicolumn{5}{|c|}{$\begin{array}{c}\text { Tabela VI - Taxas de reestenose coronariana após o implante do stent } \\
\text { ou angioplastia com o balão, em estudos randomizados com vasos } \\
\text { pequenos }(\geq 2,0 \text { até } \leq 3,0 \mathrm{~mm}) .\end{array}$} \\
\hline & \multirow{2}{*}{$\begin{array}{l}\text { Número de } \\
\text { pacientes }\end{array}$} & \multicolumn{2}{|c|}{ Reestenose } & \multirow[t]{2}{*}{ OR } \\
\hline & & Balão & $\overline{\text { Stent }}$ & \\
\hline ISAR-SMART & 406 & $37,4 \%$ & $35,7 \%$ & 1,08 \\
\hline SISA & 351 & $32,4 \%$ & $28 \%$ & 1,25 \\
\hline SISCA & 145 & $18,8 \%$ & $9,7 \%$ & 2,34 \\
\hline Be-SMART & 381 & $48,8 \%$ & $22,7 \%$ & 3,19 \\
\hline RAP & 426 & $37 \%$ & $27 \%$ & 1,61 \\
\hline COAST & 128 & $31 \%$ & $26 \%$ & 1,26 \\
\hline Total & 1.837 & $35,6 \%$ & $26,5 \%$ & 1,69 \\
\hline
\end{tabular}

A utilização sistemática de um inibidor da glicoproteína IIb/IIIa, associada ao procedimento, iniciada na sala de cardiologia invasiva, é controversa (CADILLAC e ADMIRAL) $)^{60,61}$. Seu uso fica reservado a situações especiais, como inabilidade em restabelecer o fluxo coronariano normal de maneira mecânica, grande quantidade de trombos ou tratamento de pacientes em classe funcional Killip > 2 (Classe IIa, nível de evidência $A$ ).

No entanto, sua administração como facilitador da reperfusão coronariana, previamente à admissão na sala de cardiologia invasiva, é promissora, com elevação das taxas de sucesso e melhoria da recuperação da função ventricular esquerda. Maiores séries são necessárias para fornecer uma indicação mais consistente ${ }^{62-65}$ (Classe IIb, nível de evidência $B$ ).

\section{VI - Indicações angiográficas}

A) Diâmetro do vaso - Não existem dúvidas em relação à superioridade dos resultados oferecidos com o implante dos stents coronarianos em vasos com diâmetro $\geq 3,0 \mathrm{~mm}$, evidências essas obtidas quando da comparação com o balão em estudos randomizados (tab V) $)^{66,67}$ (Classe I, nível de evidência A).

Nos vasos pequenos ( $\geq 2,0$ até $<3,0 \mathrm{~mm})$, entretanto, ainda existe controvérsia. No seguimento tardio, as taxas de reestenose são maiores, assim como a apresentação na forma oclusiva. A associação com extensão longa (> $25 \mathrm{~mm}) \mathrm{e}$ diabetes pode oferecer resultados tardios ainda mais adversos. Estudos randomizados avaliaram esse subgrupo angiográfico específico ${ }^{68-73}$. No total, 1.837 casos foram analisados, sendo que a média dos diâmetros de referência des-

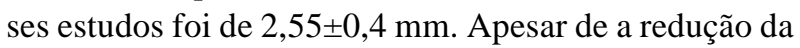
reestenose coronariana ter sido de $25 \%$, em média, a reestenose intra-stent ficou próxima de $30 \%$, quase o dobro do observado em vasos de maior calibre (tab VI). A relevância clínica da estenose, quando localizada em um vaso pequeno (área de miocárdio em risco não extensa), deve ser levada em consideração na indicação do procedimento (Classe IIB, nível de evidência $\mathbf{A}$ ). 
Arq Bras Cardiol

volume 80, (suplemento I), 2003
Diretriz de Indicações e Utilizações das Intervenções Percutâneas e Stent Intracoronariano na Prática Clínica
A nova tecnologia dos stents revestidos apresenta resultados promissores neste grupo de pacientes. Uma análise de subgrupos, realizada em dois estudos que compararam os stents recobertos com rapamicina com os convencionais (RAVEL e SIRIUS), demonstrou melhores resultados quando foram utilizados os stents recobertos, com reduções expressivas nas taxas de reestenose (RAVEL: 0\% vs. 28,9\%; $\mathrm{p}<0,001$; SIRIUS: vasos com diâmetro de 2,3mm: $1,7 \%$ vs. 32,7\%; $\mathrm{p}<0,001$; vasos com diâmetro de 2,8mm: $1,9 \%$ vs. $37,5 \%$; $<<0,001)(53,54,74)$.

B) Extensão da estenose-alvo - A extensão da lesão coronariana é um fator prognóstico independente para ocorrência de complicações imediatas durante a realização de uma angioplastia com o balão. A reestenose coronariana é superior a $50 \%$. Os stents também melhoraram os resultados nesse subgrupo de estenoses; contudo as cifras de reestenose já publicadas variam de 30 até $60 \%$, com uma relação direta entre a extensão da estenose-alvo e a reobstrução do vaso tratado ${ }^{75-79}$.

Apenas um estudo randomizado, até o momento, mereceu relevância clínica (ADVANCE). Nesse ensaio, 437 pacientes com estenoses longas (>20 até $50 \mathrm{~mm}$, média de $27 \pm 10 \mathrm{~mm}$ ) foram selecionados para o implante de stent longo ou angioplastia ótima. Observou-se que 34\% dos casos não lograram a randomização, por necessitarem do implante de um stent imediato, devido à não obtenção de um resultado ótimo com o balão ${ }^{80}$.

Apesar de os níveis de nova revascularização terem sido semelhantes (balão/stent: 14,7\% vs. 17,9\%), as taxas de reestenose foram menores com o implante dos stents $(27 \%$ vs. 42\%; $\mathrm{p}=0,022$ ). Quando possível, a sua indicação é superior à angioplastia apenas com o balão (Classe IIa, nível de evidência B)

C) Oclusão crônica - A realização de angioplastia com o balão em oclusões crônicas (> 3 meses) está associada a altas taxas de reestenose e de nova oclusão (50\% e 70\%, respectivamente). Três ensaios randomizados compararam a utilização de stent com o balão, em pacientes com oclusão crônica(SICCO, GISSOC e TOSCA). No estudo SICCO, 119 pacientes foram analisados. A reestenose e a reoclusão foram significativamente menores com o implante dos stents ( $73,7 \%$ vs. $31,6 \%$ e $26 \%$ vs. $12 \%$ ( $\mathrm{p}<0,02$ ), respectivamente). O estudo GISSOC confirmou essas reduções em 110 casos (68\% vs. $32 \%$ e $34 \%$ vs. $8 \%$ ( $\mathrm{p}<0,001)$, reestenose e reoclusão). Levando em consideração esses dados, o implante de stents é o método de escolha em oclusões crônicas, desde que elas possam ser cruzadas com o fio-guia ${ }^{81-83}$ (Classe I, nível de evidência $\mathbf{A}$ ).

Em virtude de casos de impossibilidade de ultrapassar a oclusão com o fio-guia convencional, novos dispositivos estão sendo progressivamente testados para lograr esse objetivo (Front Runner, Lumed ${ }^{\circledR}$ ). Embora ainda não estejam disponíveis para a prática clínica no Brasil, tais dispositivos representam um avanço seguro no sentido de solucionar uma das mais antigas limitações da intervenção percutânea coronariana.

D) Lesão reestenótica - D.1) Após angioplastia como balão: O implante destent é preferencial, como demonstrado pelo estudo randomizado REST, comparativo com o balão. Em 383 pacientes analisados, os stents foram superiores à nova angioplastia com o balão, reduzindo a necessidade de realizar uma nova revascularização para o vaso-alvo (10\% vs. 27\%; $\mathrm{p}=0,001)$ em decorrência da redução da reestenose (18\% vs. 32\%; $\mathrm{p}=0,03)^{84}$ (Classe I, nível de evidência B).

D.2) Intra-stent: Este tipo de reestenose é decorrente unicamente de hiperplasia mio-intimal excessiva ${ }^{85}$. Três padrões angiográficos básicos são detectados: reestenose focal, difusa e proliferativa (até a oclusão). Nas reestenoses focais ( $<10 \mathrm{~mm}$ de extensão), pode ser utilizada angioplastia com balão ou mesmo uma técnica atero-ablativa. Numa análise retrospectiva, que utilizou casos pareados tratados com

\begin{tabular}{|c|c|c|c|c|c|c|c|}
\hline Estudo & $\mathrm{R}$ & Pacientes & Isótopo & Dose (Gy) & $\begin{array}{l}\text { Extensão } \\
\text { irradiada }\end{array}$ & $\begin{array}{l}\text { Reestenose } \\
\text { clínica }\end{array}$ & $\begin{array}{l}\text { Reestenose } \\
\text { angiográfica }\end{array}$ \\
\hline SCRIPPS & Sim & 55 & $\operatorname{Ir}^{192}$ & USIC & $\leq 30 \mathrm{~mm}$ & $15 \% / 48 \% *$ & $33 \% / 64 \% *$ \\
\hline GAMMA I & Sim & 252 & $\operatorname{Ir}^{192}$ & USIC & $\leq 45 \mathrm{~mm}$ & $24 \% / 45 \% *$ & $22 \% / 51 \% *$ \\
\hline GAMMA II & Não & 125 & $\operatorname{Ir}^{192}$ & 14 & $\leq 45 \mathrm{~mm}$ & $23 \%$ & $25 \%$ \\
\hline$\beta$-WRIST & Não & 50 & $\mathrm{Y}^{90}$ & 18 & $\leq 47 \mathrm{~mm}$ & $34 \%$ & $22 \%$ \\
\hline START & Sim & 476 & $\mathrm{Sr} / \mathrm{Y}^{90}$ & $16 / 20$ & $\leq 20 \mathrm{~mm}$ & $13 \% / 23 \% *$ & $14 \% / 41 \% *$ \\
\hline START $^{40 / 20}$ & Não & 137 & $\mathrm{Sr} / \mathrm{Y}^{90}$ & $16 / 20$ & $\leq 20 \mathrm{~mm}$ & $11 \%$ & $16 \%$ \\
\hline Beta-CATH & Sim & 1456 & $\mathrm{Sr} / \mathrm{Y}^{90}$ & $14 / 18$ & $\leq 20 \mathrm{~mm}$ & $13 \% / 15 \%$ & $31 \% / 36$ \\
\hline PREVENT & Sim & 105 & $\mathrm{P}^{32}$ & $16 / 20 / 24$ & $\leq 25 \mathrm{~mm}$ & $6 \% / 24 \% *$ & $8 \% / 39 \% *$ \\
\hline INHIBIT & Sim & 332 & $\mathrm{P}^{32}$ & 20 & $\leq 47 \mathrm{~mm}$ & $11 \% / 29 \% *$ & $26 \% / 52 \% *$ \\
\hline GENEVA & Sim & 181 & $\mathrm{Y}^{90}$ & $9 / 12 / 15 / 18$ & $\leq 15 \mathrm{~mm}$ & $6 \%$ (18Gy) & $4 \%$ (18Gy) \\
\hline
\end{tabular}


o balão cortador ou angioplastia com balão, foi observado sucesso elevado em ambos os grupos e taxas de nova recorrência em $25 \%$ dos pacientes tratados com o balão cortador e em até $45 \%$ no grupo do balão convencional ${ }^{86}$. Entretanto, estudos comparativos diretos entre angioplastia convencional e técnicas atero-ablativas ( rotablator e balão cortador) não mostraram superioridade destas sobre o balão no tratamento da reestenose intra-stent ${ }^{87,88}$. (Classe IIa, nível de evidência B).

A braquiterapia intracoronariana, com raios gama ou beta, apresenta-se como a técnica que proporciona os melhores resultados tardios (tab VII), principalmente nos pacientes diabéticos, demonstrando os menores níveis de nova reestenose. Contudo, o procedimento requer maior complexidade, relacionada ao cuidado necessário para aplicação intra-hospitalar de radiação gama ou beta ${ }^{89,90}$. Além disto, complicações tardias têm sido relatadas, arrefecendo a indicação dessa modalidade terapêutica (Classe I, nível de evidência $A$ ).

Existe a possibilidade de estender os bons resultados obtidos com o implante dos stents revestidos com fármacos antiproliferativos para o tratamento da reestenose intra-stent. Uma única série já foi apresentada, envolvendo 25 pacientes consecutivos com reestenose intra-stent, tratados, em São Paulo, com o implante dostent com siroli$m u$ s. No seguimento de um ano, apenas um caso apresentou nova reestenose ${ }^{91}$.

E) Enxerto de veia safena - A causa mais comum de isquemia miocárdica, após a realização da cirurgia de revascularização, é a degeneração ateromatosa do enxerto de veia safena. A angioplastia coronariana com o balão não exibiu, em séries históricas, bons resultados, principalmente quando do tratamento de estenoses de localização ostial ou daquelas degeneradas no corpo do enxerto.

Poucos estudos compararam o balão ou o stent nesse subgrupo angiográfico, talvez pela dificuldade de randomizar pacientes para a técnica do balão, após o advento dos stents. A maior série (SAVED) incluiu 220 casos, obtendo maiores níveis de sucesso inicial nos pacientes que receberam stent (92\% vs. 69\%; $\mathrm{p}<0,001$ ), com diminuição da ocorrência dos eventos maiores combinados (óbito, infarto do miocárdio e nova ATC) aos oito meses (26\% vs. 39\%; $\mathrm{p}=0,04)$, mas sem redução significante da reestenose angiográfica (37\% vs. 46\%; $\mathrm{p}=0,24)(92)$.

Uma nova complicação foi observada, porém, com o progressivo implante de stents: o deslocamento para a circulação distal de fragmentos aterotrombóticos, provenientes da lesão-alvo submetida ao implante da endoprótese, ocasionando, em até $30 \%$ dos casos, oclusão e interrupção do fluxo coronariano distal (no reflow), com surgimento de infarto do miocárdio. Muitas vezes, a complicação é imprevisível. Os enxertos venosos mais antigos ( $\geq 5$ anos), com importante degeneração (>25 mm), associados à redução do fluxo coronariano epicárdico (TIMI<3), antes mesmo da realização do procedimento, devem alertar o operador para sua ocorrência ${ }^{92,93}$.

Os sistemas de filtros de proteção distal foram desenvolvidos para superar essa complicação, encontrando-se já disponíveis. Um estudo randomizado comparou o implante de stent com ou sem proteção distal por meio de filtro (SAFER). Foram selecionados 801 pacientes e a média das idades dos enxertos tratados foi superior a dez anos. Aqueles que realizaram o procedimento com o auxílio do filtro (GuardWire ${ }^{\circledR}$ ) obtiveram maior cifra de fluxo TIMI-3 (98\% vs. $95 \%$; $\mathrm{p}=0,04)$ e redução de $50 \%$ na ocorrência do fenômeno no reflow ( $3 \%$ vs. $9 \%$; $\mathrm{p}=0,001)$. Conseqüentemente, a utilização do filtro também reduziu, significativamente, o diagnóstico de infarto do miocárdio após o implante do stent $(8,6 \%$ vs. $14,7 \%$; $\mathrm{p}=0,008)(93)$.

A recomendação atual é de que o implante de stent em enxertos de veia de safena deva ser realizado com auxílio de filtro de proteção distal, principalmente em estenoses com as características já descritas. O implante do stent em estenoses ostiais ou na anastomose distal pode prescindir do uso de filtros (Classe IIa, nível de evidência B).

É, contudo, contra-indicada, na prática atual, a realização de revascularização percutânea com uso do stent em um enxerto degenerado, que supre grande área de miocárdio viável, sem a devida proteção da circulação coronariana distal (Classe III, nível de evidência A).

F) Lesão em bifurcação - A intervenção coronariana percutânea de estenoses em bifurcação permanece um desafio. Os resultados observados com o balão ( $k$ issingballoon) são pobres. As técnicas atero-ablativas de execução complexa e o implante de stents coronarianos de primei-

\begin{tabular}{|c|c|c|c|c|c|c|c|}
\hline \multirow[t]{2}{*}{ Estudo } & \multirow[t]{2}{*}{ Pacientes } & \multicolumn{2}{|c|}{ Infarto } & \multicolumn{2}{|c|}{ Morte } & \multicolumn{2}{|c|}{ Nova Revascularização } \\
\hline & & Stent & Cirurgia & Stent & Cirurgia & Stent & Cirurgia \\
\hline ARTS & 1205 & $6,2 \%$ & $4,8 \%$ & $2,5 \%$ & $2,8 \%$ & $16,8 \%$ & $3,5 \%$ \\
\hline ERACI-II & 450 & $2,3 \%$ & $6,6 \%$ & $3,1 \%$ & $7,5 \%$ & $16,8 \%$ & $4,8 \%$ \\
\hline SOS & 967 & nd & nd & $2,5 \%$ & $0,8 \%$ & $22 \%$ & $5,8 \%$ \\
\hline
\end{tabular}


Arq Bras Cardiol

volume 80, (suplemento I), 2003
Diretriz de Indicações e Utilizações das Intervenções Percutâneas e Stent Intracoronariano na Prática Clínica ra geração também ofereceram dificuldades técnicas. O desenvolvimento de novos stents, mais flexíveis, com hastes que possibilitem sua dilatação, e o implante de umstent adicional para o ramo adjacente aprimoraram os resultados e expandiram essa indicação, sendo mais freqüente sua utilização nos dias atuais ${ }^{94-97}$. Inúmeras técnicas foram idealizadas e apresentadas, visando a contornar a grande variação morfológica observada nas estenoses localizadas em bifurcação. Essa diversidade de apresentações dificulta uma recomendação objetiva. A presença de estenose no ramo adjacente, seu calibre ( $<3,0 \mathrm{~mm} v s$. $>3,0 \mathrm{~mm})$, o ângulo do ramo em relação ao vaso principal são as principais variantes. Talvez por este motivo, acrescido ao fato de observarem-se maus resultados com a técnica do balão, nenhum estudo controlado tenha sido realizado até o momento. As séries consecutivas demonstram cifra de sucesso elevado, porém a reestenose intra-stent, em um ou em ambos os vasos acometidos na bifurcação, é superior àquela observada com o implante de um único stent.

A recomendação atual está relacionada ao diâmetro do ramo adjacente. Nas estenoses em bifurcação, com o ramo adjacente também apresentando estenose grave e de calibre $>3,0 \mathrm{~mm}$, o tratamento cirúrgico deve ser considerado. Se a opção for pela abordagem percutânea, o consenso atual, baseado na experiência de centros com grande experiência no tratamento desses casos, propõe implante de stent no ramo principal e realização de angioplastia convencional com stent provisional no ramo secundário. Por tratarse de uma área ainda controversa, a intervenção coronariana percutânea em estenoses bifurcadas deve ser individualizada, com cuidadosa análise anatomofuncional, associada à experiência do operador com esta apresentação morfológica, que é fundamental para o sucesso do procedimento ${ }^{94-97}$ (Classe IIb, nível de evidência C).

G) Intervenção percutânea multiarterial - No passado, seis trabalhos multicêntricos e randomizados compararam, em pacientes com doença multiarterial, os resultados da angioplastia com o balão e os da cirurgia de revascularização, demonstrando que ambas as possibilidades de tratamento exibiam resultados tardios similares, em termos de sobrevida e taxas de infarto do miocárdio. Nos casos em que foi realizada a angioplastia com o balão, houve maior número de novas revascularizações, com significativo retorno dos sintomas anginosos, sendo que os diabéticos exibiram maior mortalidade ao final de sete anos de evolução $0^{98,99}$.

Atualmente, estão disponíveis novos estudos randomizados, comparando o implante de múltiplos stents (> um vaso epicárdico) e a cirurgia de revascularização (tab VIII) ${ }^{100-102}$.

A ocorrência de infarto do miocárdio e de óbito ao final de um ano apresenta diferenças de maneira até oposta entre os estudos, possivelmente justificadas pela demografia e técnica utilizadas. No entanto, a maior necessidade de repetir um novo procedimento de revascularização nos pacientes submetidos ao implante destents foi unânime.

Recomenda-se a escolha criteriosa de casos para o tratamento percutâneo em múltiplos vasos. Portadores de doença biarterial, com estenoses focais e função contrátil do ventrículo esquerdo preservada, parecem ser os melhores candidatos, assim como aqueles em situação oposta, com alto risco cirúrgico (morbidades associadas) ou impossibilidade de utilizar anastomose de artéria mamária ${ }^{99}$ (Classe I, nível de evidência A). Especial atenção deve ser dada à seleção do procedimento de revascularização a ser empregado em pacientes diabéticos com doença multiarterial, visto que, mesmo nos ensaios clínicos nos quais foram utilizados stents coronarianos, a evolução do grupo cirúrgico foi mais favorável. Desta forma, nos diabéticos tratados (com insulina ou hipoglicemiante orais) que podem receber, pelo menos, um implante de mamária, o tratamento cirúrgico ainda deve ser considerado preferencial.

\section{VII- Considerações finais}

A evolução dos dispositivos percutâneos tem sido rápida e com incorporação imediata, visto a freqüente realização de ensaios controlados, fornecendo recomendações consistentes. O futuro da intervenção coronariana percutânea é promissor. Os resultados imediatos foram melhorados com a adoção dos stents coronarianos. A divulgação das conclusões dos ensaios RAVEL e SIRIUS é desafiadora, podendo ampliar, ainda mais, o espectro de pacientes e de lesões passíveis de serem submetidas ao método ${ }^{53,54}$. Nesses estudos, após o implante de stents com sirolimus, foram obtidas baixas taxas de reestenose intra-stent $(<2 \%)$, de maneira segura, sem a ocorrência de trombose, formação de aneurismas ou outras complicações maiores.

\section{Referências}

1. Grüntzig AR, Senning A, Siegenthaler WE. Nonoperative dilatation of coronary-artery stenosis: percutaneous transluminal coronary angioplasty. N Engl J Med 1979;301:61-8.

2. Anderson HV, Shaw RE, Brindis RG, e cols. Cardiology-national cardiovascular data registry (ACC-NCDR). J Am Coll Cardiol 2002;39:1096-103.
3. Sousa AGMR, Mattos LA, Moura Campos Neto C, et al. Intervenções percutâneas para revascularização do miocárdio no Brasil em 1996 e 1997 comparadas às do biênio 1992-93. Relatório do registro CENIC (Central Nacional de Intervenções Cardiovasculares). Arq Bras Cardiol 1998;70:423-30.

4. Sigwart U, Puel J, Mircovitcht V, et al. Intravascular stents to prevent o0cclu- 
sion and restenosis after trasnluminal angioplasty. N Eng J med 1987; 316:701-9.

5. Serruys PW, De Jaegere P, Kiemeneij F, et al. Comparison of balloon expandable stent implantation with balloon angioplasty in patients with coronary artery disease. N Engl J Med 1994;331:489-95.

6. Fischman DL, Leon MB, Baim D, et al. A randomized comparison of coronary stent placement and balloon angioplasty in the treatment of coronary artery disease. N Engl J Med 1994;331:496-01.

7. Serruys PW, van Hout B, Bonier H, et al. A randomized comparison of implantation of heparin coated stents with balloon angioplasty in selected patients with coronary artery disease (BENESTENT II). Lancet 1998;352:673-81.

8. Smith SC, Dove JT, Jacobs AK, et al. ACC/AHA guidelines of percutaneous coronary interventions (revision of the 1993 PTCA guidelines)-executive summary A report of the American College of Cardiology/American Heart Association Task Force on Practice Guidelines (Committee to revise the 1993 guidelines for percutaneous transluminal coronary angioplasty). J Am Coll Cardiol 2001;37: 2215-38.

9. Stone GW, Mehran R, Dangas G, et al. Differential impact on survival of electrocardiographic Q-wave versus enzymatic myocardial infarction after percutaneous intervention: a device-specific analysis of 7147 patients. Circulation 2001;104:642-7.

10. Holmes DR, Berger PB. Troponisms, necrosettes, enzyme leaks, creatinine phosphokinase bumps, and infarctlets: what's behind this new lexicon and what does it add? Circulation 2001;104:627-9.

11. Cantor WJ, Newby LK, Christenson RH, et al. Prognostic significance of elevated troponin I after percutaneous coronary intervention. J Am Coll Cardiol 2002;39:1738-44.

12. II Guidelines of the Brazilian Cardiology Society for acute myocardial infarct treatment. July 22 to 25 of 1999. Teresópolis-RJ. Arq Bras Cardiol 2000;74 (suppl 2):1-46.

13. Aversano T, Aversano LT, Passamani E, et al. Thrombolytic therapy vs primary percutaneous coronary intervention for myocardial infarction in patients presenting to hospitals without on-site cardiac surgery: a randomized controlled trial. JAMA 2002;287:1943-51.

14. Vakili BA, Kaplan R, Brown DL. Volume-outcome relation for physicians and hospitals performing angioplasty for acute myocardial infarction in New York state. Circulation 2001;104:2171-6.

15. Grines CL, Westerhausen DR, Grines LL, et al. A randomized trial of transfer for primary angioplasty versus on-site thrombolysis in patients with high-risk myocardial infarction: the Air Primary Angioplasty in Myocardial Infarction study. J Am Coll Cardiol 2002;39:1713-9.

16. Singh M, Ting HH, Berger PB, Garratt KN, Holmes DR, Gersh BJ. Rationale for on-site cardiac surgery for primary angioplasty: a time for reappraisal. J Am Coll Cardiol 2002;39:1881-9.

17. Mattos LA, Sousa AG, Campos Neto C, et al. Primary coronary angioplasty and a stent implantation in acute myocardial infarction. Comparative analysis of the in-hospital results in the CENIC/SBHCI registry. National Center of Cardiovascular Interventions members. Arq Bras Cardiol 1999;73:475-84

18. Mattos LA, Sousa AGMR, Pinto IMF, et al. on behalf of the CENIC investigators (Central Nacional de Intervenções Cardiovasculares)/SBHCI (Sociedade Brasileira de Hemodinâmica e Cardiologia Intervencionista). Primary coronary angioplasty in 9,434 patients during acute myocardial infarction: predictors of inhospital major adverse events during 1996 to 2000 in Brazil. Arq Bras Cardiol 2002;78 [No prelo]

19. Colombo A, Hall P, Nakamura S, et al. Intracoronary without anticoagulation accomplished with intravascular ultrasound guidance. Circulation 1995;91: 1676-88.

20. Schomig A, Neumann FJ, Kastrati A, et al. A randomized comparison of antiplatelet and anticoagulation therapy after the placement of coronary artery stents. N Engl J Med 1996;334:1084-9.

21. Leon MB, Baim DS, Popma JJ, et al. A clinical trial comparing three antithrombotic drug regimens after coronary stenting. N Engl J Med 1998;339:1665-71.

22. Baim DS. PREDICT: a prospective multicenter randomized trial of stent implantation with versus without pre dilatation. TCT 2000, Washington D.C. USA October 2000.

23. Brito FS Jr, Caixeta AM, Perin MA, et al. Comparison of direct stenting versus stenting with predilation for the treatment of selected coronary narrowings. Am J Cardiol 2002;89:115-20.

24. Gruberg L, Pinnow E, Flood R, et al. Incidence, management, and outcome of coronary artery perforation during percutaneous coronary intervention. Am J Cardiol 2000;86:680-2.
25. Pijls NH, Klauss V, Siebert U, et al. Coronary pressure measurement after stenting predicts adverse events at follow-up: a multicenter registry. Circulation. 2002;105:2950-4.

26. Pijls NHJ, De Bruyne B, Peels K, et al. Measurement of fractional flow reserve to assess the functional severity of coronary artery stenoses. N Engl J Med. 1996; 334: 1703-1708

27. Van Liebergen RAM, Piek JJ, Koch KT, de Winter RJ, Lie KI. Immediate and long-term effect of balloon angioplasty or stent implantation on the absolute and relative coronary blood flow velocity reserve. Circulation 1998; 98 : 2133-2140.

28. Fearon WF, Luna J, Samady H, et al. Fractional flow reserve compared with intravascular ultrasound guidance for optimizing stent deployment. Circulation. $2001 ; 104: 1917-22$.

29. Nakamura S, Hall P, Gaglione A, et al. High pressure assisted coronary stent implantation accomplished without intravascular ultrasound guidance and subsequent anticoagulation. J Am Coll Cardiol 1997;29:21-7.

30. Frei AW, Roskman H, Hodgson JM, et al. IVUS-guided stenting: does acute angiography predict long term outcome? Insights from the strategy of IVUS-guided PTCA and stenting, SIPS. Circulation 1997:96;1222-28.

31. Nicosia A, Russo RJ, Yock PG, et al. IVUS findings in angiographically optimized stents in native vessels and vein grafts: Lessons from the AVID study (Angiography versus intravascular ultrasound direct stent placement). J Am Coll Cardiol 1997;29:280A (abstract)

32. Albiero R, Rau T, Schluter M, et al. Comparison of immediate and intermediate term results of intravascular ultrasound versus angiography guided PalmazSchatz stent implantation in matched lesions. Circulation 1997;96:2997-05.

33. Fitzgerald PJ, Oshima A, Hayase M, et al. Final results of the CRUISE (Can Routine Ultrasound Influence Stent Expansion). Circulation 2000;102:523-30.

34. Mudra H, di Mario C, de Jaegere $\mathrm{P}$, et al. Randomized comparison of coronary stent implantation under ultrasound or angiographic guidance to reduce stent restenosis (OPTICUS Study). Circulation 2001;104:1343-9.

35. Bhatt DL, Bertrand ME, Berger PB, et al. Meta-analysis of randomized and registry comparisons of ticlopidine with clopidogrel after stenting. J Am Coll Cardiol 2002;39:9-14.

36. Mehta SR, Yusuf S, Peters RJ, et al. Effects of pretreatment with clopidogrel and aspirin followed by long-term therapy in patients undergoing percutaneous coronary intervention: the PCI-CURE study. Lancet 2001;358:527-33.

37. Lincoff AM, Califf RM, Topol EJ. Platelet glycoprotein IIb/IIIa receptor blockade in coronary artery disease. J Am Coll Cardiol 2000;35:1103-15.

38. O'Shea JC, Buller CE, Cantor WJ, et al. Long-term efficacy of platelet glycoprotein IIb/IIIa integrin blockade with eptifibatide in coronary stent intervention. JAMA 2002;287:618-21.

39. Kereiakes DJ, Anderson KM, Achenbach RE, et al. Abciximab survival advantage is not explained by reduction in early major cardiac events: EPIC, EPILOG and EPISTENT 3-Year analysis. Circulation 2001;104:II-87 (abstract).

40. Quinn MJ, Plow EF, Topol EJ. Platelet glycoprotein IIb/IIIa inhibitors: recognition of a two-edged sword? Circulation 2002;106:379-85.

41. Serruys PW, de Feyter P, Macaya C, et al. Fluvastatin for prevention of cardiac events following successful first percutaneous coronary intervention. A randomized controlled trial. JAMA 2002:287:3215-3222

42. Trial of invasive versus medical therapy in elderly patients with chronic symptomatic coronary-artery disease (TIME): a randomised trial. Lancet 2001;358:951-7.

43. Kornowski R, Mintz G, Kent KM, et al. Increased restenosis in diabetes mellitus after coronary interventions is due to exaggerated intimal hyperplasia. A serial intravascular study. Circulation 1997;335:217-25.

44. Kastrati A, Schomig A, Elezi S, et al. Predictive factors of restenosis after coronary artery stent placement. J Am Coll Cardiol 1997;30:1428-36.

45. Van Belle E, Bauters C, Hubert E, et al. Restenosis rates in diabetic patients: a comparison of coronary stenting and balloon angioplasty in native coronary vessels. Circulation 1997;96:1454-60.

46. Abizaid A, Kornowski R, Mintz G, et al. The influence of diabetes mellitus on acute and late clinical outcomes following coronary stent implantation. J Am Coll Cardiol 1998;32:584-9.

47. Elezi S, Kastrati A, Pache J, et al. Diabetes Mellitus and the clinical and angiographic outcome after coronary stent placement. J Am Coll Cardiol 1998; 32:1866-73.

48. Van Belle E, Ketelers R, Bauters C, et al. Patency of percutaneous transluminal coronary angioplasty sites at 6-month angiographic follow-up. A key determinant of survival in diabetics after coronary balloon angioplasty. Circulation 2001; 103:1218-24.

49. Chaves AJ, Mattos LA, Pinto I, et al. A less favorable long-term outcome in diabe- 
tics persists despite treatment with intracoronary stents. J Am Coll Cardiol 2000;35:55A (abstract).

50. Mattos L, Grines CL, Sousa JE, et al. One-year clinical follow-up after primary coronary interventions for acute myocardial infarction in diabetic patients: a substudy from the Stent PAMI trial. Arq Bras Cardiol 2001;77:556-61.

51. Lincoff AM, Califf RM, Moliterno DJ, et al. Complementary clinical benefits of coronary-artery stenting and blockade of platelet glycoprotein IIb/IIIa receptors. N Engl J Med 1999;341:319-27.

52. Abizaid A, Costa MA, Centemero M, et al. Clinical and economic impact of diabetes mellitus on percutaneous and surgical treatment of multivessel coronary disease patients: insights from the Arterial Revascularization Therapy Study (ARTS) trial. Circulation 2001;104:533-8.

53. Sousa JE, Costa MA, Abizaid A. et al. Sustained suppression of neointimal proliferation by sirolimus-eluting stents: one-year angiographic and intravascular ultrasound follow-up. Circulation 2001;104:2007-11.

54. Morice MC, Serruys PW, Sousa JE, et al. A randomized comparison of a sirolimuseluting stent with a standard stent for coronary revascularization. N Engl J Med 2002;346:1773-80.

55. Wallentin L, Lagerqvist B, Husted S, Kontny F, Stahle E, Swahn E. Outcome at 1 year after an invasive compared with a non-invasive strategy in unstable coronary-artery disease: the FRISC II invasive randomised trial. FRISC II Investigators (Fast Revascularisation during Instability in Coronary artery disease). Lancet 2000;356:9-16

56. Cannon CP, Weintraub WS, Demopoulos LA, et al. Comparison of early invasive and conservative strategies in patients with unstable coronary syndromes treated with the glycoprotein IIb/IIIa inhibitor tirofiban. N Engl J Med 2001; 344:1879-87.

57. Stone GW, Grines CL, Browne KF, et al. Implications of recurrent ischemia after reperfusion therapy in acute myocardial infarction: a comparison of thrombolytic therapy and primary angioplasty. J Am Coll Cardiol 1995;26:66-72.

58. Grines CL, Cox D, Stone GW, et al. Coronary angioplasty with or without stent implantation for acute myocardial infarction. N Engl J Med 1999; 341:1949-56

59. Mattos LA, Grines CL, Cox D, et al. A comparative analysis of primary stenting and optimal balloon coronary angioplasty in acute myocardial infarction. Six month results from the Stent PAMI trial. Arq Bras Cardiol 2000;75:499-514.

60. Stone GW, Grines CL, Cox D, et al. A prospective randomized trial comparing balloon angioplasty with or without abciximab to primary stenting with or without abciximab in acute myocardial infarction. N Engl J Med 2002; 346:957-66.

61. Montalescot G, Barragan P, Wittenberg O, et al. Platelet glycoprotein IIb/IIIa inhibition with coronary stenting for acute myocardial infarction. N Engl J Med 2001;344:1895-903.

62. Antman EM, Giugliano RP, Gibson CM, et al. Abciximab facilitates the rate and extent of thrombolysis: results of the thrombolysis in myocardial infarction (TIMI) 14 trial. The TIMI 14 Investigators. Circulation 1999;99:2720-32.

63. Neumann FJ, Blasini R, Schmitt C, et al. Effect of glycoprotein IIb/IIIa receptor blockade on recovery of coronary flow and left ventricular function after the placement of coronary-artery stents in acute myocardial infarction. Circulation 1998;98:2695-701.

64. Neumann FJ, Kastrati A, Schmitt C, et al. Effect of glycoprotein IIb/IIIa receptor blockade with abciximab on clinical and angiographic restenosis rate after the placement of coronary stents following acute myocardial infarction. J Am Coll Cardiol 2000;35:915-21.

65. Van den Merkhof LF, Zijlstra F, Olsson H, et al. Abciximab in the treatment of acute myocardial infarction eligible for primary percutaneous transluminal coronary angioplasty. Results of the Glycoprotein Receptor Antagonist Patency Evaluation (GRAPE) pilot study. J Am Coll Cardiol 1999;33:1528-32.

66. Keane D, Azar AJ, de Jaegere P, et al. Clinical and angiographic outcome of elective stent implantation in small coronary vessels: an analysis of the BENESTENT trial. Semin Interv Cardiol 1996;1:255-62.

67. Elezi S, Kastrati A, Neumann FJ, et al. Vessel size and long-term outcome after coronary stent placement. Circulation 1998;98:1875-80.

68. Savage MP, Fiscman DL, Kada F, et al. A randomized comparison of elective stenting and balloon angioplasty of small coronary arteries. Circulation 1999;100: I-503 (abstract)

69. Kastrati A, Schomig A, Dirschinger J, et al. A randomized trial comparing stenting with balloon angioplasty in small vessels in patients with symptomatic coronary artery disease. ISAR-SMART Study Investigators. Intracoronary Stenting or Angioplasty for Restenosis Reduction in Small Arteries. Circulation 2000;102:2593-8.
70. Koning R, Eltchaninoff $\mathrm{H}$, Commeau P, et al. Stent placement compared with balloon angioplasty for small coronary arteries: in-hospital and 6-month clinical and angiographic results. Circulation 2001;104:1604-8.

71. Doucet S, Schalij MJ, Vrolix MC, et al. Stent placement to prevent restenosis after angioplasty in small coronary arteries. Circulation 2001;104:2029-33.

72. Moer R, Myreng Y, Molstad P, et al. Stenting in small coronary arteries (SISCA) trial. A randomized comparison between balloon angioplasty and the heparincoated beStent. J Am Coll Cardiol 2001;38:1598-603.

73. Garcia E, Gomez-Recio M, Moreno R, et al. Stent reduces restenosis in small vessels: results of the RAP study. J Am Coll Cardiol 2001;37:17A (abstract).

74. A Multicenter double blind study of the SIRolImUS-Coated BX Velocity Balloon-expandable stent in the treatment of patients with de novo coronary artery lesions. http://www.tctmd.com.

75. Kornowski R, Mehran R, Lansky AJ, et al. Procedural results and late clinical outcomes following percutaneous interventions using long ( $\geq 25 \mathrm{~mm}$ ) stents. J Am Coll Cardiol 1998;33:69A (abstract).

76. Kobayashi Y, De Gregorio J, Reimers B, et al. The length of the stent segment is an independent predictor of restenosis. J Am Coll Cardiol 1999;33:366A (abstract).

77. Elezi S, Kastrati A, Wehinger A, et al. Stent placement in long $(\geq 15 \mathrm{~mm})$ coronary lesions. J Am Coll Cardiol 1998;33:273A (abstract).

78. Nakagawa Y, Yufu K, Tamura T, et al. Stenting for long lesions with long GFX stent. J Am Coll Cardiol 1999;33:69A (abstract).

79. O emrawsingh $P$, Schalij M, Van der Wall E. Guidance of long stent implantation by intravascular ultrasound has a significantly better outcome compared to implantation guided by angiography alone: final results of a randomized study (TULIP). J Am Coll Cardiol 2001;37:20A (abstract).

80. Serruys PW, Foley DP, Suttorp MJ, et al. A randomized comparison of the value of additional stenting after optimal balloon angioplasty for long coronary lesions: final results of the additional value of NIR stents for treatment of long coronary lesions (ADVANCE) study. J Am Coll Cardiol 2002;39:393-9.

81. Sirnes PA, Golf S, Myreng Y, et al. Sustained benefit of stenting chronic coronary occlusion: long-term clinical follow-up of the stenting in chronic coronary occlusion (SICCO) study. J Am Coll Cardiol 1998; 32:305-10.

82. Rubartelli P, Niccoli L, Verna E, et al. Stent implantation versus balloon angioplasty in chronic coronary occlusions: results from the GISSOC trial. Gruppo Italiano di Studio sullo Stent nelle Occlusioni Coronariche. J Am Coll Cardiol 1998; 32:90-6.

83. Buller CE, Dzavik V, Carere RG, et al. Primary stenting versus balloon angioplasty in occluded coronary arteries: the Total Occlusion Study of Canada (TOSCA). Circulation 1999;100:236-42.

84. Erbel R, Haude M, Hopp HW, et al. Coronary-artery stenting compared with balloon angioplasty for restenosis after initial balloon angioplasty. Restenosis Stent Study Group. N Engl J Med 1998;339:1672-8.

85. Hoffmann R, Mintz GS, Dussaillant GR, et al. Patterns and mechanisms of in stent restenosis. A serial intravascular ultrasound tudy. J Am Coll Cardiol 1996;28:1134-39.

86. Adamian M, Colombo A, Briguori C, et al. Cutting balloon angioplasty for the treatment of in-stent restenosis: a matched comparison with rotational atherectomy, additional stent implantation and balloon angioplasty. J Am Coll Cardiol 2001;38:672-9.

87. Vom Dahl J, Dietz U, Philipp Haager K, et al. Rotational atherectomy does not reduce recurrent in-stent restenosis: results of the angioplasty versus rotational atherectomy for treatment of diffuse in-stent restenosis trial (ARTIST) Circulation, 2002;105:583-8.

88. Albiero R. The Restenosis Cutting Balloon Evaluation (RESCUT). TCT 2002, Washington DC, USA.

89. Bhargava B, Tripuraneni P. Role of intracoronary brachytherapy for in-stent restenosis. Lancet 2002; 359:543-4.

90. Brito FS Jr, Hanriot R, Almeida BO, et al. Intracoronary brachytherapy in the treatment of in-stent restenosis. Initial experience in Brazil.Arq Bras Cardiol. 2001;77:266-73

91. Abizaid A, Serruys PW, Abizaid A, et al. The absence of edge effect after implantation of sirolimus-eluting stents to treat in-stent restenosis: a three-dimensional intravascular ultrasound volumetric anaylsis. J Am Coll Cardiol 2002;39: 58A (abstract).

92. Savage MP, Douglas JS, Fischman DL, et al. Stent placement compared with balloon angioplasty for obstructed coronary bypass grafts. Saphenous Vein De Novo Trial Investigators. N Engl J Med 1997;337:740-7.

93. Baim DS, Wahr D, George B, et al. Randomized trial of a distal embolic protection device during percutaneous intervention of saphenous vein aorto-coronary bypass grafts. Circulation 2002;105:1285-90. 
94. Yamashita T, Nishida T, Adamian MG, et al. Bifurcartion lesions: two stents versus one stent: immediate and follow-up results. J Am Coll Cardiol 2000;35:45-51.

95. Lefevre T, Louvard Y, Morice MC, Loubeyre C, Piechaud JF, Dumas P. Stenting of bifurcation lesions: a rational approach. J Interv Cardiol 2001;14:573-85.

96. Sheiban I, Albiero R, Marsico F, et al. Immediate and long-term results of "T" stenting for bifurcation coronary lesions. Am J Cardiol 2000;85:1141-4 A9.

97. Gobeil F, Lefevre T, Guyon P, et al. Stenting of bifurcation lesions using the Bestent: a prospective dual-center study. Catheter Cardiovasc Interv 2002;55:427-33.

98. The Bypass Angioplasty Revascularization Investigation (BARI) Investigators: Seven-year outcome in the bypass angioplasty revascularization investigation (BARI) by treatment and diabetic status. J Am Coll Cardiol 2000;35 1112-9.
99. Taggart DP, D'Amico R, Altman DG. Effect of arterial revascularisation on survival: a systematic review of studies comparing bilateral and single internal mammary arteries. Lancet 2001;358:870-5.

100. Results from late-breaking clinical trials sessions at ACC 2001. J Am Coll Cardiol;38:595-612.

101. Rodriguez A, Bernardi V, Navia J, et al. Argentine Randomized Study: Coronary Angioplasty with Stenting versus Coronary Bypass Surgery in patients with Multiple-Vessel Disease (ERACI II): 30-day and one-year follow-up results. ERACI II Investigators. J Am Coll Cardiol 2001; 37:51-8.

102. Serruys PW, Unger F, Sousa JE, et al. Comparison of coronary-artery bypass surgery and stenting for the treatment of multivessel disease. N Engl J Med $2001 ; 344: 1117-24$. 